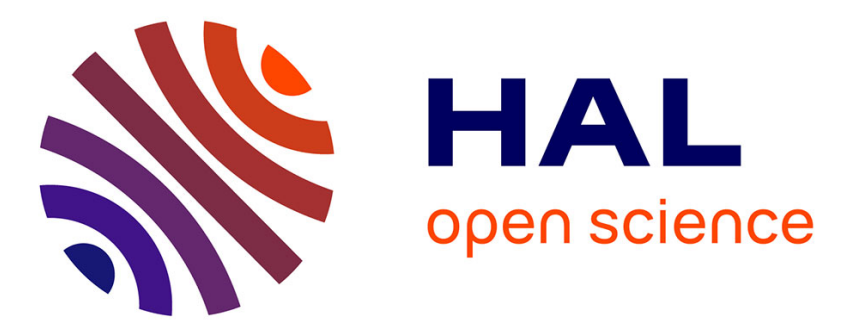

\title{
Lifetimes of atoms trapped in an optical lattice in proximity of a surface
}

Sophie Pelisson, Riccardo Messina, Marie-Christine Angonin, Peter Wolf

\section{To cite this version:}

Sophie Pelisson, Riccardo Messina, Marie-Christine Angonin, Peter Wolf. Lifetimes of atoms trapped in an optical lattice in proximity of a surface. Physical Review A : Atomic, molecular, and optical physics [1990-2015], 2013, 88 (1), pp.013411. 10.1103/PhysRevA.88.013411 . hal-01334978

\section{HAL Id: hal-01334978 \\ https://hal-iogs.archives-ouvertes.fr/hal-01334978}

Submitted on 21 Jun 2016

HAL is a multi-disciplinary open access archive for the deposit and dissemination of scientific research documents, whether they are published or not. The documents may come from teaching and research institutions in France or abroad, or from public or private research centers.
L'archive ouverte pluridisciplinaire HAL, est destinée au dépôt et à la diffusion de documents scientifiques de niveau recherche, publiés ou non, émanant des établissements d'enseignement et de recherche français ou étrangers, des laboratoires publics ou privés. 


\title{
Lifetimes of atoms trapped in an optical lattice in proximity of a surface
}

\author{
Sophie Pelisson, ${ }^{1, *}$ Riccardo Messina, ${ }^{1,2}$ Marie-Christine Angonin, ${ }^{1}$ and Peter Wolf ${ }^{1}$ \\ ${ }^{1}$ LNE-SYRTE, Observatoire de Paris, CNRS UMR8630, UPMC, 61 avenue de l'Observatoire, F-75014 Paris, France \\ ${ }^{2}$ Laboratoire Charles Fabry, Institut d'Optique, CNRS, Université Paris-Sud, Campus Polytechnique, \\ RD128, F-91127 Palaiseau Cedex, France
}

(Received 14 February 2013; published 15 July 2013)

\begin{abstract}
We study the lifetime of an atom trapped in an optical vertical lattice in proximity of a massive surface using a complex-scaling approach. We analyze how the presence of the surface modifies the known lifetimes of Wannier-Stark states associated with Landau-Zener tunneling. We also investigate how the existence of a hypothetical short-distance deviation from Newton's gravitational law could affect these lifetimes. Our study is relevant in order to discuss the feasibility of any atomic-interferometry experiment performed near a surface. Finally, the difficulties encountered in applying the complex-scaling approach to the atom-surface Casimir-Polder interaction are addressed.
\end{abstract}

DOI: 10.1103/PhysRevA.88.013411

PACS number(s): 37.10.Jk, 34.35.+a, 37.25.+k, 42.50.Ct

\section{INTRODUCTION}

The problem of atoms trapped in an accelerated periodic lattice is an old one in solid-state physics [1-5]. However, some phenomena like pseudoeigenstates (Wannier-Stark ladders) and the lifetime limitations of those states (Landau-Zener tunneling) are still a current subject of research both on the experimental side and theoretically [6-13] (see also [5] and references therein for a more extensive review). In this work, we study the Landau-Zener effect [14-16] for atoms trapped in a vertical standing wave in the presence of short-scale interactions.

This is done in the context of the theoretical modeling of the experiment "Force de Casimir et Gravitation à courte distance" (FORCA-G) aiming at measuring the CasimirPolder force acting on a neutral atom in the presence of a massive dielectric surface by atomic interferometry. At the same time, the experiment is configured to search for non-Newtonian deviations from the gravitational law at short distances [17-23]. These two goals are reached by the use of atoms trapped in a vertical optical standing wave in front of a massive surface realizing the trap. Such a lattice allows a precise knowledge of the distance between the atom and the surface, this distance being a multiple of the wavelength of the trap. The atomic states in such a trap were the main subject of a previous paper [19]. In that paper the shape of the wave functions and the modification of energy levels due to the presence of the surface were deduced by solving a standard eigenvalue problem. In this context, the states are supposed to be bound states of the system, meaning that their lifetime is infinite.

Nevertheless, solid-state physics teaches us that the states in an accelerated periodic lattice are not bound states but resonance states with a finite lifetime. The subject of this paper is to quantify these lifetimes for the states calculated in [19] in order to check the feasibility of the experiment, knowing that the measurement of the interaction by atom interferometry takes at most a few seconds. We want to understand, in more detail, how the presence of the surface

\footnotetext{
*sophie.pelisson@obspm.fr
}

and of a hypothetical non-Newtonian gravitational potential modifies the well-known finite lifetimes of standard WannierStark states.

This paper is organized as follows. In Sec. II, we describe the system under scrutiny and the method we use to calculate the lifetime of the atomic states. Section III is devoted to the effect of the surface. This section is separated in two parts. As a first step, we study the effect of a surface treated as a boundary condition for an atom below it. In the following, we investigate the phenomena arising when tunneling of the atom through the surface is allowed. This is done for an atom above the surface in order to clearly discriminate the effect of the finiteness of the potential barrier from other resonance effects. Then, Sec. IV shows the effect of a deviation from Newton's gravitational law at a short distance on the lifetimes of the trapped atom, and Sec. V discusses the problem arising when the Casimir-Polder interaction is taken into account. In this part, the difficulties of the treatment of the Casimir-Polder effect by the complex-scaling method are highlighted.

\section{LIFETIMES OF ATOMS IN WANNIER-STARK STATES}

Let us consider the Hamiltonian of an atom of mass $m_{a}$ trapped in a periodic lattice $V(z)=\frac{U}{2}\left[1-\cos \left(2 k_{l} z\right)\right]$ in the presence of a linear gravitational potential

$$
H_{\mathrm{WS}}=\frac{p^{2}}{2 m_{a}}+\frac{U}{2}\left[1-\cos \left(2 k_{l} z\right)\right]-m_{a} g z,
$$

where $U$ is the depth of the trapping potential, $k_{l}$ represents the wave vector of the trap, and $g$ is the earth's gravitational constant. This operator is the sum of the well-known Bloch Hamiltonian $H_{B}=\frac{p^{2}}{2 m_{a}}+\frac{U}{2}\left[1-\cos \left(2 k_{l} z\right)\right][1]$ and a linear potential $-m_{a} g z$ (the $z$ axis is oriented downwards). As a consequence, the eigenstates of Eq. (1) are derived from the Bloch states, generally taking into account one single Bloch band. The spectrum obtained using this procedure can be written as

$$
E_{\alpha, n}=\bar{\varepsilon}_{\alpha}-n m_{a} g \frac{\lambda_{l}}{2},
$$

where $\frac{\lambda_{l}}{2}$ is the periodicity of the lattice, with $\lambda_{l}$ being its wavelength, $\bar{\varepsilon}_{\alpha}$ is the mean value of the energy of the Bloch 
band $\alpha$ under consideration, and $n$ is an integer labeling the well where the state is centered. The states obtained are known as Wannier-Stark states and were first discussed for electrons in a crystal submitted to a constant electric field [2]. The existence of such eigenstates results from the single-band approximation. Nevertheless, a calculation ignoring this approximation confirms the existence of these states but associates a finite lifetime to each of them. This lifetime physically corresponds to the so-called Landau-Zener tunneling, namely, the possibility for a particle in a WannierStark lattice site to transit from one Bloch band to another [14-16]. In other words, the discrete spectrum in Eq. (2) is immersed in the continuum of eigenvalues of the Hamiltonian (1) and can thus be seen as a resonance spectrum [24]. The spectrum of Eq. (2) should thus be rewritten as [3-5]

$$
E_{\alpha, n}=\bar{\varepsilon}_{\alpha}-n m_{a} g \frac{\lambda_{l}}{2}-i \frac{\Gamma_{\alpha}}{2} .
$$

The eigenstates associated with the spectrum (3) are metastable states with a finite lifetime $\tau=\frac{\hbar}{\Gamma_{\alpha}}$. In order to completely characterize the states of an atom in our optical lattice, we need to work out this complex spectrum to evaluate their lifetime in the trap.

Several methods have been developed so far in order to study this complex spectrum [25-30], each using approximations such as a periodic potential with a finite number of gaps or a finite periodic lattice. Most of these methods are based on the analogy of the Wannier-Stark sates with scattering states. In this theoretical framework, the complex spectrum (3) can be calculated as the set of poles of the scattering matrix of the system. The method we will use in this work is based on the rotation of the Hamiltonian (1) in the complex plane and the derivation of the complex spectrum as eigenvalues of the obtained non-Hermitian Hamiltonian. This approach is known as complex-scaling method [3,31,32], and the rotation of the Hamiltonian is performed via the operator $C(\theta)$ such that

$$
C(\theta) \varphi(z)=\varphi\left(z e^{i \theta}\right) \quad \forall \varphi \in C_{0}^{\infty} .
$$

The one-dimensional complex-scaled Hamiltonian is then

$$
H_{\mathrm{WS}}^{(\theta)}=\frac{e^{-2 i \theta} p^{2}}{2 m_{a}}+\frac{U}{2}\left[1-\cos \left(2 k_{l} z e^{i \theta}\right)\right]-m_{a} g z e^{i \theta} .
$$

According to the Balslev-Combes theorem [33], the complex spectrum (3) can then be found by solving the eigenvalue problem

$$
H_{\mathrm{WS}}^{(\theta)}\left|\phi_{\alpha, n}\right\rangle=E_{\alpha, n}\left|\phi_{\alpha, n}\right\rangle,
$$

where the real part of a given eigenvalue represents its energy level, while its imaginary part is the width of the resonance.

We stress here that resonance wave functions are characterized by a divergent behavior when $z \rightarrow \infty$ as

$$
\phi_{\text {res }}(z \rightarrow \infty) \simeq e^{-i k z}+S(k) e^{i k z},
$$

with $k$ being the wave vector of the atom and $S(k)$ being the scattering matrix of the system. As demonstrated in [3], the divergent part of the resonant wave functions in Eq. (7) is regularized by the complex scaling. This is not the case for a nonresonant wave function of the continuum. As a consequence, it is important to distinguish the resonant from the nonresonant part of the complex spectrum worked out

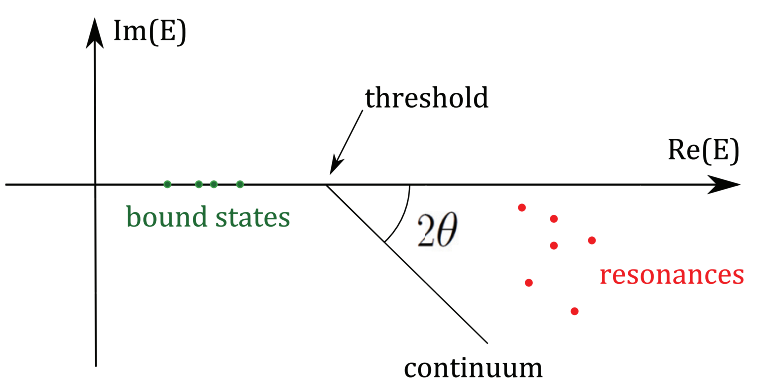

FIG. 1. (Color online) Schematic representation of the complex eigenvalues of the complex-coordinate scaled Hamiltonian $H^{(\theta)}$ according to the Balslev-Combes theorem [33].

solving Eq. (6). A schematic representation of the eigenvalues of Hamiltonian (5) is shown in Fig. 1. We see in Fig. 1 that the continuum eigenvalues lie on a line forming an angle $2 \theta$ with the real axis, with $\theta$ being the angle chosen for the transformation (4). At the same time the real bound states are located on the real axis (corresponding to the fact that they have an infinite lifetime), while the resonance states are the complex eigenvalues which are not part of the rotated continuum. In the case of the Wannier-Stark Hamiltonian, the presence of energy bands due to the periodicity of the trap leads to a peculiar structure of the complex eigenvalues. The eigenvalues of the Hamiltonian (5) are shown in Fig. 2. These eigenvalues are obtained using the pseudoperiodicity of the trap. Indeed, the fact that we calculate states in an infinite pseudoperiodic lattice allows us to restrict the search of the spectrum in Eq. (3) to the analysis of the spectral properties of the Floquet-Bloch operator [5]

$$
U=e^{-\frac{i}{\hbar} H_{\mathrm{Ws}} T_{B}}, \quad T_{B}=\frac{2 \hbar}{m_{a} g \lambda_{l}},
$$

over a Bloch period $[4,5]$. The eigenvalues are then worked out using a discrete variable representation (DVR) method and a QR algorithm for complex symmetric matrices [34].

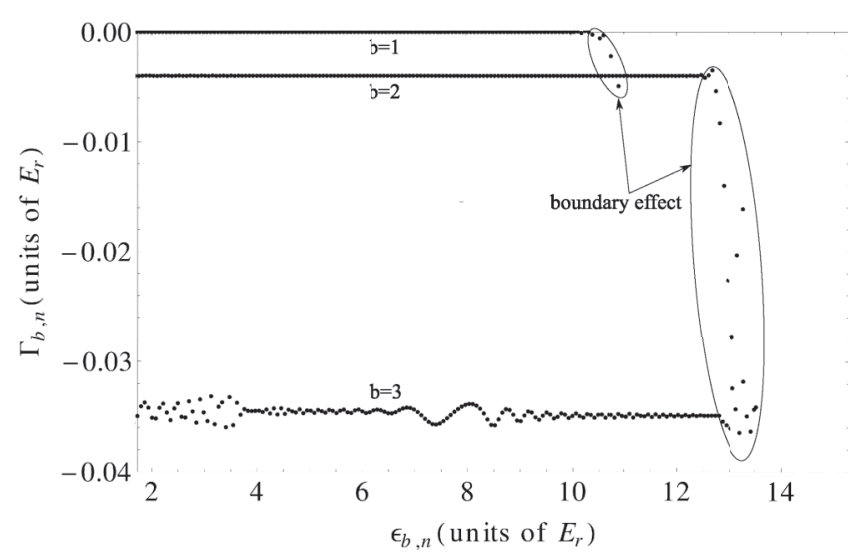

FIG. 2. Complex eigenvalues for the first three Bloch bands of the Hamiltonian $H_{\mathrm{ws}}^{(\theta)}$. The energies are expressed in units of $E_{r}=$ $\frac{\hbar^{2} k_{l}^{2}}{2 m_{a}}$, which is the natural unit for energy levels in a trap with wave vector $k_{l}$ (in the case of FORCA-G, $E_{r} \simeq 5.37 \times 10^{-30} \mathrm{~J}$ and $\Gamma=$ $1 E_{r}$ corresponds to $\left.\tau=\hbar / \Gamma=1.96 \times 10^{-5} \mathrm{~s}\right)$. The spectrum here is calculated for $U=3 E_{r}$. 
TABLE I. Table of the real parts $\operatorname{Re}\left(E_{1, n}\right)$ of the complex energies (3) compared with the Wannier-Stark spectrum $\mathcal{E}_{1, n}$ obtained numerically without complex scaling and with the energies $\mathcal{E}_{n}$ resulting from a semianalytical calculation in the first Bloch band. The energies are given in units of $E_{r}=\frac{\hbar^{2} k_{l}^{2}}{2 m_{a}}$, which is the natural unit for energy levels in a trap with a wave vector $k_{l}$. The numerical example corresponds to $U=3 E_{r}$.

\begin{tabular}{lccc}
\hline \hline$n$ & $\operatorname{Re}\left(E_{1, n}\right)$ & $\mathcal{E}_{1, n}$ & $\mathcal{E}_{n}$ \\
\hline-5 & 1.78711 & 1.78711 & 1.78718 \\
-4 & 1.71703 & 1.71703 & 1.71711 \\
-3 & 1.64696 & 1.64696 & 1.64704 \\
-2 & 1.57688 & 1.57688 & 1.57697 \\
-1 & 1.50681 & 1.50681 & 1.50690 \\
0 & 1.43674 & 1.43674 & 1.43683 \\
1 & 1.36667 & 1.36667 & 1.36677 \\
2 & 1.29660 & 1.29660 & 1.29670 \\
3 & 1.22652 & 1.22652 & 1.22663 \\
4 & 1.15645 & 1.15645 & 1.15656 \\
5 & 1.08638 & 1.08638 & 1.08649 \\
\hline \hline
\end{tabular}

In order to check the validity of our method, we compare the real parts of the calculated eigenvalues with the spectrum obtained using two different methods. The first is the tightbinding model [5,17], a standard semianalytical resolution of the Schrödinger equation for $H_{\mathrm{WS}}$, which does not give access to the finite lifetime of the states. The second one is a finite-difference numerical approach to $H_{\mathrm{Ws}}$ without complex scaling. Table I shows good agreement between the real parts of eigenvalues (3) and the second approach. The difference between our results and the tight-binding model can be traced back to the fact that this approach restricts the Hilbert space to the first Bloch band.

Concerning the imaginary parts, representing the lifetime of the band under scrutiny, Niu et al. have proposed a general formula to evaluate the inverse lifetime of the standard Wannier-Stark states [35]. This formula gives an estimate of the tunneling rate $\gamma$ as a function of a critical acceleration $\alpha_{c}$ and the dimensionless acceleration $\alpha$ due to the linear potential.

$$
\gamma=\alpha e^{-\alpha_{c} / \alpha} \text {. }
$$

In our case, $\alpha=\frac{m_{a}^{2} g}{\hbar^{2} k_{l}^{3}}$ and the critical acceleration can be expressed as

$$
\alpha_{c}=\frac{\pi \Delta^{2}}{K}
$$

where $\Delta$ is the half width of the energy gap between the first and the second Bloch bands and $K=\frac{n}{2}$ is the wave number of Bragg scattering corresponding to the $n$th gap (here we will take $n=1$ ). With this expression, we obtain, for the second Bloch band, a tunneling rate of the order of $0.003 E_{r}$, which is of the same order of magnitude as the value of the complex part of the spectrum (3), as can be seen in Fig. 2. An additional verification with other values of the well depth assures us that the complex-scaling method provides satisfying results for this physical system. As a consequence, in the next section, we will use this method to analyze the lifetimes of the Wannier-Stark states in the presence of a surface, whose main features have been already discussed in [19].

\section{LIFETIMES IN THE PRESENCE OF THE SURFACE}

\section{A. Presence of the surface}

In our problem, we consider the presence of a surface at $z=0$. Our atom is thus located below the surface, in the region $z>0$ (we recall that the $z$ axis is oriented downwards). The presence of this boundary condition breaks the quasiperiodicity of the system. The potential modifying the optical trap is no longer linear since it must be considered the gravitational linear potential for $z>0$ and an infinite potential barrier for $z \leqslant 0$, describing the impossibility of the particle to penetrate the mirror. We have shown in a previous paper [19] that this surface induces a modification of the energies and states of the Hamiltonian $H_{\text {Ws }}$. We now want to verify that this modification does not reduce drastically the lifetimes of our atoms in the trap.

As anticipated before, the presence of a boundary condition in $z=0$ breaks the translational symmetry of the problem. Thus we can no longer use the quasiperiodic approximation, and we have to solve directly the eigenvalues problem (6). This is done using a finite-element method and once again a QR algorithm for symmetric complex matrices using the subroutine ZGEEV of the numerical package LAPACK [36]. However, using this method, numerical problems arise when the well depth is larger than $U=2.2 E_{r}$ since in this case the imaginary parts of the complex eigenvalues are too small. As a matter of fact, the lifetime of a given Wannier-Stark well is a strongly increasing function of the well depth. This dependence can be well understood using the image of tunneling. Indeed, the finite lifetime of the metastable states can be seen as a consequence of the possibility for an atom in a lattice site $n$ to tunnel through the potential toward an upper Bloch band in another site. This is favored by the linear potential which induces resonant tunneling between the first band in a given site to, e.g., the second band in a farther site. As a consequence the atom reaches a new state with a new energy, destroying the former one. Moreover, the atom can reach a state with an energy higher than the trap depth, thus no longer being trapped. So the tunneling probability depends on the lattice depth: the deeper the trap is, the weaker this probability becomes.

This feature is illustrated in Fig. 3, where lifetimes are calculated for different well depths. Here we have taken into account only the lifetime of the first Bloch band. Indeed, as can be seen in Fig. 2, the lifetime of the first band is the longest one, and the larger the band under scrutiny is, the shorter this lifetime is. In the particular case of our experimental parameters, the second Bloch band has a mean energy of $\bar{\epsilon}_{2} \simeq 5.45 E_{r}$ and is then not trapped by a well depth $U=3 E_{r}$. As a consequence, only the lifetime of the first band is of interest to us.

The analysis of Fig. 3 shows, moreover, that for any well depth, the first-band lifetimes are a function of the considered well. In particular, the lifetimes do not vary remarkably starting approximately five wells away from the surface, while being functions of the considered well in proximity of the boundary $z=0$. In order to verify the coherence of the calculation the constant lifetimes far from the surface (function of the well depth) can be compared to the ones obtained through the Landau-Zener formula, derived for the standard Wannier-Stark states (i.e., in an infinite lattice). This comparison is shown in Fig. 4. In Fig. 4, we can see that the Landau-Zener formula 


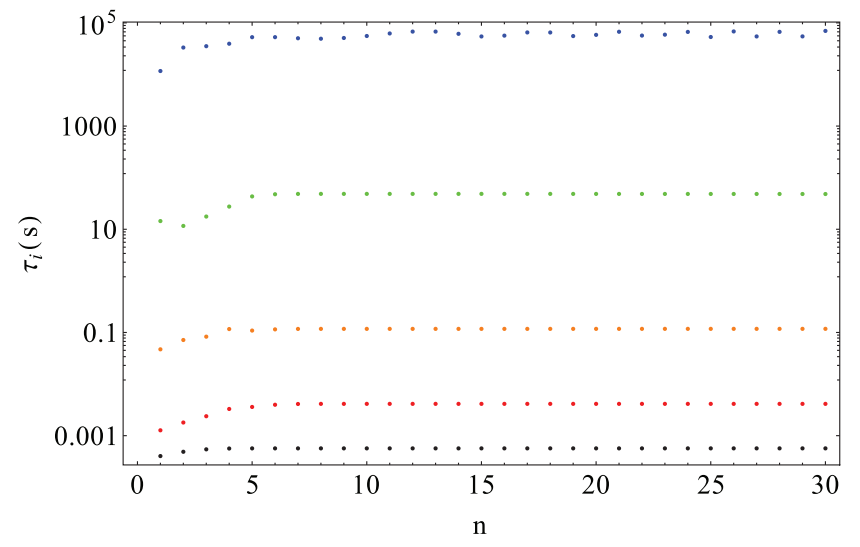

FIG. 3. (Color online) Lifetimes of the first Bloch band as a function of the quantum number $n$ labeling the well of the trap for five different depths. From top to bottom we have $U=2 E_{r}$ (blue dots), $U=1.5 E_{r}$ (green dots), $U=1 E_{r}$ (orange dots), $U=0.5 E_{r}$ (red dots), and $U=0.1 E_{r}$ (black dots).

still gives a good description far from the surface. We observe typical oscillations of lifetimes around the Landau-Zener results, which have already been discussed theoretically in [5] and observed experimentally in [8]. These oscillations result from resonant tunneling occurring for specific values of $U$ between the most stable band and excited ones (for a detailed discussion see [5]). This suggests that the lifetimes of our metastable states in the presence of a surface are of the order of $10^{14} \mathrm{~s}$ for a well depth of $U=3 E_{r}$. However, in this analysis we have treated the mirror as an infinite barrier at $z=0$, which is not very realistic. It is of interest to investigate how the results are modified if the mirror is modeled by means of a finite potential barrier. This will be done assuming that the atom is located above the mirror.

\section{B. Atom above the mirror}

A way to increase the lifetime of the states is to place the atom above the surface rather than below it. In this case, if we consider as before the mirror as an infinite barrier, the potential is a well of infinite height. As a consequence, it supports only a discrete set of real eigenstates having an infinite lifetime.

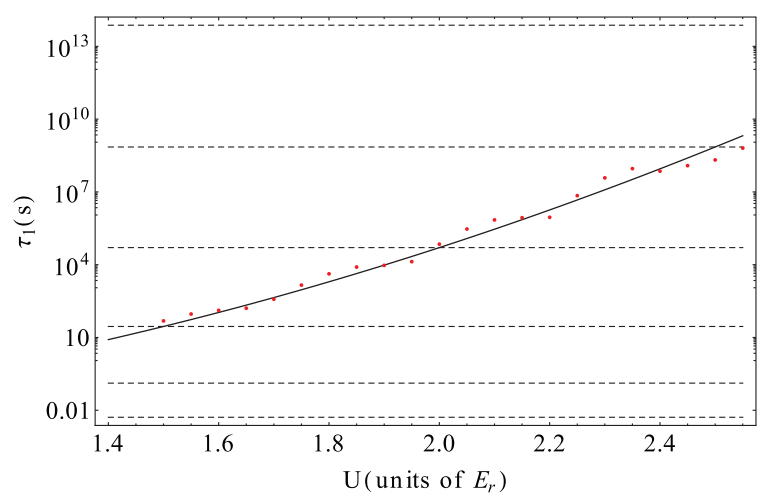

FIG. 4. (Color online) Lifetime of the first Bloch band calculated with the complex scaling method (red dots) as a function of the trap depth. The calculated lifetimes are compared with the Landau-Zener formula (black line) for standard Wannier-Stark sates.

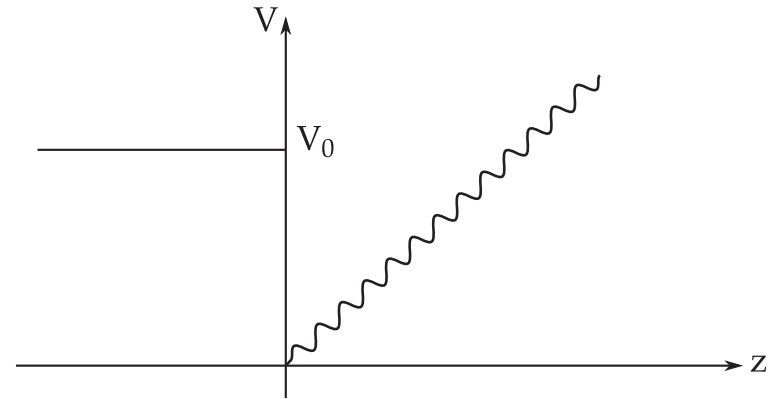

FIG. 5. Shape of the potential for atoms above a finite surface.

In order to model the surface more precisely, we allow the tunneling of the atom into this surface. Therefore we replace the infinite barrier by a finite one as shown in Fig. 5. As a consequence, the complex-scaled Hamiltonian of the system can be expressed as

$$
\begin{aligned}
H_{\mathrm{above}}^{(\theta)}= & \frac{e^{-2 i \theta} p^{2}}{2 m_{a}}+V_{0} \quad \text { if } z \leqslant 0, \\
H_{\mathrm{above}}^{(\theta)}= & \frac{e^{-2 i \theta} p^{2}}{2 m_{a}}+\frac{U}{2}\left[1-\cos \left(2 k_{l} z e^{i \theta}\right)\right] \\
& +m_{a} g z e^{i \theta} \quad \text { elsewhere, }
\end{aligned}
$$

where $V_{0} \in \mathbb{R}$. In order to study the behavior of the atoms in front of a surface with a finite size, we investigate the complex spectrum for a given well depth $\left(U=1 E_{r}\right.$ in the numerical example) as a function of the height of the barrier chosen to represent the surface. More specifically, we focus our attention on the transition from bound states to resonances for atoms in front of a potential barrier with a varying height.

The resulting complex eigenvalues for different heights of the barrier are shown in Fig. 6. In Fig. 6, we see that the presence of the barrier induces the emergence of a continuum associated with the standard resonances spectrum. The position of this continuum depends on the height of the barrier: more precisely, the continuum region of the spectrum always starts at a value around $V_{0}$. When the barrier is very low (i.e., of the order of the depth of the periodic trap or below), we observe that the lifetime of the atom close to this barrier is shorter than the lifetime at longer distances. This can be understood thanks to the interpretation of Landau and Zener of the resonance phenomenon. Indeed, if we consider that the finite lifetime of the atom arises from the resonant tunneling through the trap, it is clear that this tunneling has a higher probability when the atom is close to the barrier because it is not trapped on the left side. In addition, we observe that when the barrier becomes higher than the well depth, some bound states appear that correspond to the closest states submitted to a potential well.

As expected, the effect of a finite barrier representing the surface is completely negligible for the wells far from the surface and starts to be visible for the closest wells when the barrier height is of the order of the well depth or below. This means that the penetrability of the surface should be comparable to that of the periodic trap in order to allow a significant modification of the behavior of the complex spectrum. This is reasonably not at all the case for a solid mirror, and we can conclude that the finiteness of the surface should not play a major role in the lifetime of our states in the trap. 

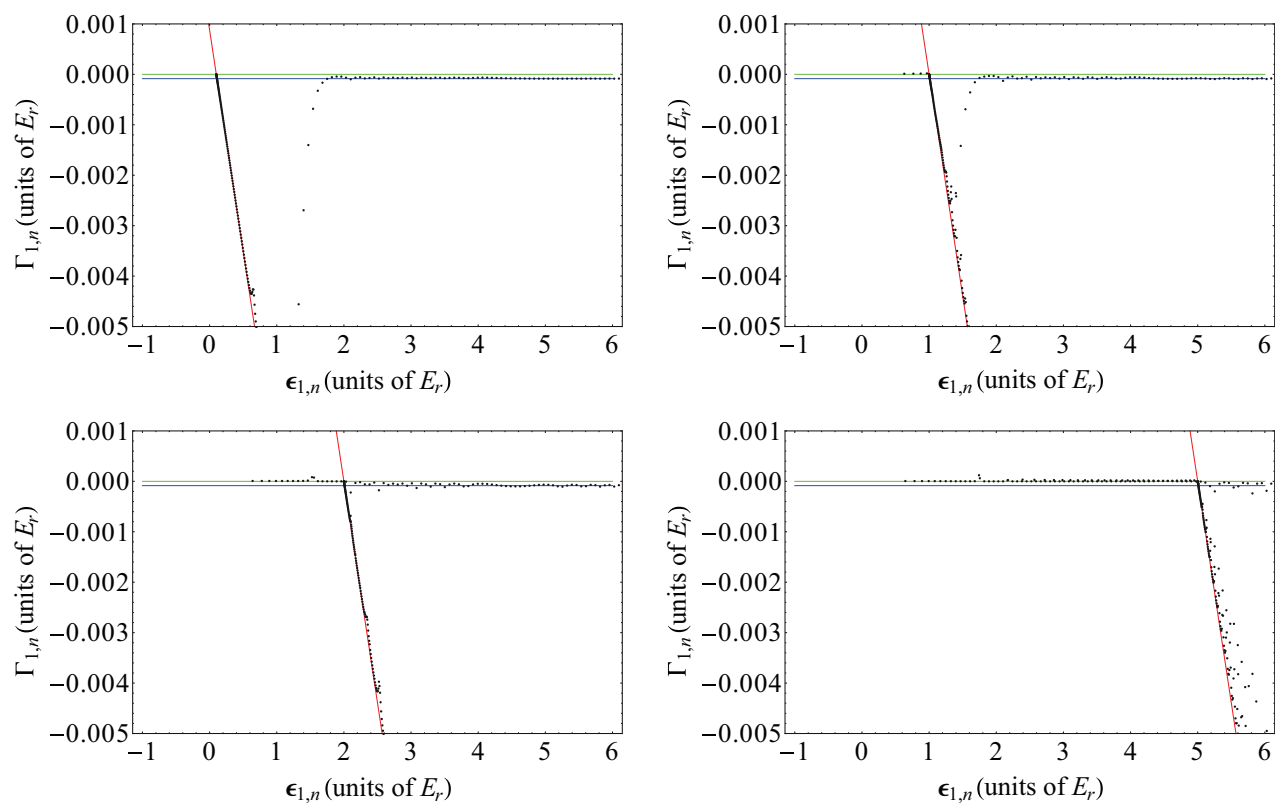

FIG. 6. (Color online) Complex spectrum of the Hamiltonian (11) describing an atom in front of a potential barrier with different heights (from left to right and top to bottom $V_{0}=0.1 E_{r}, V_{0}=1 E_{r}, V_{0}=2 E_{r}$, and $V_{0}=5 E_{r}$; black dots) compared with the standard Wannier-Stark lifetimes (blue lower horizontal line). The depth of the periodic trap is chosen to be $U=E_{r}$. The solid red line represents the line of the continuum with a slope of $2 \theta$, and the green upper horizontal line highlights the eigenvalues with an imaginary part equal to 0 .

\section{NON-NEWTONIAN GRAVITATION}

After observing that the presence of the surface does not reduce drastically the lifetime of our modified Wannier-Stark states, we have to verify that this is not the case even in the presence of a Yukawa gravitational potential. Indeed, the main goal of the experiment FORCA-G is to search for a hypothetical deviation from Newton's law at short distance predicted by some unification theories. We have then to estimate the modification of the lifetimes of the states in the presence of such a deviation, which can be written as an additional potential of the form [19]

$$
U_{\mathrm{Y}}=2 \pi \alpha_{\mathrm{Y}} G \rho_{\mathrm{s}} m_{a} \lambda_{\mathrm{Y}}^{2} e^{-2 z / \lambda_{\mathrm{Y}}},
$$

where $G=6.67 \times 10^{-11} \mathrm{~m}^{3} \mathrm{~kg}^{-1} \mathrm{~s}^{-2}$ is the universal gravitational constant and $\rho_{\mathrm{s}}$ is the density of our surface (for which we have chosen the density of silicon $\rho_{\mathrm{s}}=2.33 \times$ $10^{3} \mathrm{~kg} \mathrm{~m}^{-3}$ ). We want to stress here that the Yukawa potential presented in Eq. (12) depends on two parameters, $\alpha_{\mathrm{Y}}$ and $\lambda_{\mathrm{Y}}$. These parameters represent respectively the coupling strength of the non-Newtonian deviation and its typical range. The aim of experiments devoted to non-Newtonian gravitation is to impose constraints on the value of these two parameters. The present constraints and the ones predicted for the experiment FORCA-G are shown in Fig. 7.

In order to analyze the modification to the lifetimes induced by a hypothetical Yukawa term, we have to calculate the spectrum of the Hamiltonian

$$
\begin{aligned}
H= & H_{\mathrm{WS}}+U_{\mathrm{Y}} \\
= & \frac{p^{2}}{2 m_{a}}+\frac{U}{2}\left[1-\cos \left(2 k_{l} z\right)\right]-m_{a} g z \\
& +2 \pi \alpha_{\mathrm{Y}} G \rho_{\mathrm{s}} m_{a} \lambda_{\mathrm{Y}}^{2} e^{-2 z / \lambda_{\mathrm{Y}}} .
\end{aligned}
$$

As done previously, we apply the transformation (4) to this Hamiltonian, obtaining the non-Hermitian Hamiltonian

$$
\begin{aligned}
H^{(\theta)}= & \frac{e^{-2 i \theta} p^{2}}{2 m_{a}}+\frac{U}{2}\left[1-\cos \left(2 k_{l} z e^{i \theta}\right)\right] \\
& -m_{a} g z e^{i \theta}+2 \pi \alpha_{\mathrm{Y}} G \rho_{\mathrm{S}} m_{a} \lambda_{\mathrm{Y}}^{2} e^{-2 z e^{i \theta} / \lambda_{\mathrm{Y}}} .
\end{aligned}
$$

The complex spectrum resulting from this modified Hamiltonian is presented in Fig. 8, which shows that even if the presence of a deviation from Newton's gravitation law

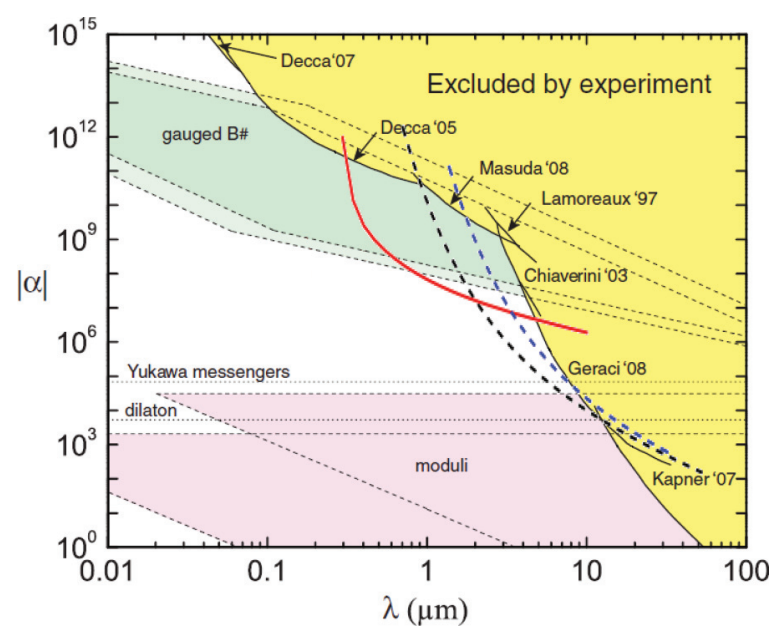

FIG. 7. (Color online) Present constraints (yellow region) and predicted constraints [colored (gray) lines] for the FORCA-G experiment for the Yukawa potential in the $\left(\alpha_{Y}, \lambda_{Y}\right)$ plane. The colored (shaded) zones are excluded. This figure is taken from [19] and adapted from [37]. 


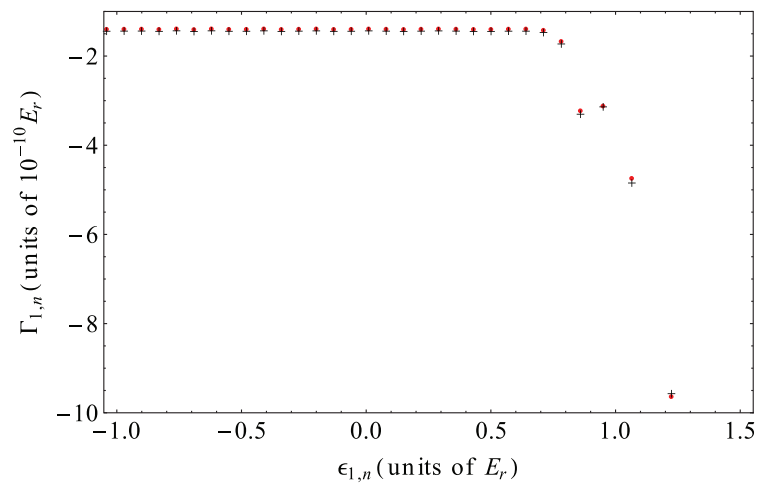

FIG. 8. (Color online) Values of the complex spectrum in the presence of a Yukawa potential (red dots) compared with the modified Wannier-Stark complex spectrum (black crosses). We have chosen $U=2 E r, \alpha_{\mathrm{Y}}=10^{11}$, and $\lambda_{\mathrm{Y}}=1 \mu \mathrm{m}$.

modifies the lifetimes of the atom in the trap, particularly for the closest wells, this lifetime remains very large compared with the duration of the experiment. Indeed, for practical reasons due to the coherence of the cold atoms in the experimental setup, the duration of the measurement in FORCA-G will not exceed $1 \mathrm{~s}$. As a consequence, the Landau-Zener effect is not a limitation for the measurement of a deviation from Newton's law in our experiment. This can be more accurately verified from the analysis in Table II, which confirms that the lifetimes of an atom in the trap are much longer than the duration of the measurement estimated in [22].

TABLE II. The first ten imaginary parts of the complex energy in the first well as a function of the values of $\alpha_{\mathrm{Y}}$ and $\lambda_{\mathrm{Y}}$ calculated in [19]. The notation $a[b]$ in the second column corresponds to $a \times 10^{b}$.

\begin{tabular}{|c|c|c|c|}
\hline $\begin{array}{l}\lambda_{\mathrm{Y}} \\
(\mu \mathrm{m})\end{array}$ & $\alpha_{\mathrm{Y}}$ & $\begin{array}{c}\operatorname{Im}\left(E_{1,1}^{(\mathrm{Y})}\right) \\
\text { (units of } 10^{-10} E_{r} \text { ) }\end{array}$ & $\begin{array}{c}\tau^{(\mathrm{Y})} \\
\left(10^{10} \mathrm{~s}\right)\end{array}$ \\
\hline \multicolumn{4}{|c|}{ Far regime } \\
\hline 0.70000 & $1.95007[12]$ & -6.25618 & 6.13778 \\
\hline 0.80835 & $2.08378[11]$ & -8.09505 & 7.94185 \\
\hline 0.93347 & $2.87073[10]$ & -8.44136 & 8.28161 \\
\hline 1.07795 & $4.92177[9]$ & -8.51022 & 8.34916 \\
\hline 1.24480 & $1.02282[9]$ & -8.52701 & 8.36564 \\
\hline 1.43747 & $2.51801[8]$ & -8.53137 & 8.36991 \\
\hline 1.65996 & $7.15759[7]$ & -8.53326 & 8.37177 \\
\hline 1.91689 & $2.30712[7]$ & -8.53361 & 8.37211 \\
\hline 2.21359 & $8.33762[6]$ & -8.53419 & 8.37268 \\
\hline 2.55622 & $3.31836[6]$ & -8.53403 & 8.37252 \\
\hline \multicolumn{4}{|c|}{ Near regime } \\
\hline 0.10000 & $9.98047[11]$ & -8.55444 & 8.39255 \\
\hline 0.11659 & $9.98047[11]$ & -8.55682 & 8.39488 \\
\hline 0.13594 & $9.98047[11]$ & -8.55945 & 8.39746 \\
\hline 0.15849 & $9.98047[11]$ & -8.56119 & 8.39917 \\
\hline 0.18479 & $9.98047[11]$ & -8.56046 & 8.39845 \\
\hline 0.21544 & $9.98047[11]$ & -8.55548 & 8.39357 \\
\hline 0.25119 & $9.98047[11]$ & -8.54216 & 8.38050 \\
\hline 0.29286 & $9.98047[11]$ & -8.51542 & 8.35427 \\
\hline 0.34146 & $1.41907[10]$ & -8.53314 & 8.37165 \\
\hline 0.39811 & $2.60450[9]$ & -8.53385 & 8.37235 \\
\hline
\end{tabular}

\section{DISCUSSION}

As we have seen in the previous sections, the presence of a material surface and the assumptions of a deviation from the short-range gravitational law do not considerably limit the lifetimes of the metastable states of the trap. As a consequence, the assumption consisting of considering these states as pseudoeigenstates remains valid under these assumptions. However, up to now, we have not taken into account the effect of the Casimir-Polder interaction between the quantized electromagnetic field in the presence of the surface and the atom. As we have seen in [19], this effect is dominant at short distances, inducing an important correction to the real energy levels of the atomic states. So we can suspect that it could have a non-negligible effect on the imaginary part of the spectrum as well.

As shown in [19], the Casimir-Polder atom-surface interaction can be computed using second-order perturbation theory on the Hamiltonian interaction term. Using the same notation as [19], the Hamiltonian describing our system can be expressed as

$$
\begin{aligned}
H & =H_{0}+H_{\mathrm{int}}=H_{\mathrm{f}}+H_{\mathrm{at}}+H_{\mathrm{WS}}+H_{\mathrm{int}}, \\
H_{\mathrm{f}} & =\sum_{p} \int_{0}^{+\infty} d k_{z} \int d^{2} \mathbf{k} \hbar \omega a_{p}^{\dagger}\left(\mathbf{k}, k_{z}\right) a_{p}\left(\mathbf{k}, k_{z}\right), \\
H_{\mathrm{at}} & =\hbar \omega_{0}|e\rangle\langle e| \\
H_{\mathrm{WS}} & =\frac{p^{2}}{2 m_{a}}-m_{a} g z+\frac{U}{2}\left[1-\cos \left(2 k_{l} z\right)\right], \\
H_{\mathrm{int}} & =-\boldsymbol{\mu} \cdot \mathcal{E}(\mathbf{r}) .
\end{aligned}
$$

The complete Hamiltonian is written as a sum of a term $H_{0}$ describing the free evolution of the atomic and field degrees of freedom. In particular, $H_{\mathrm{f}}$ is the Hamiltonian of the quantum electromagnetic field, described by a set of modes $\left(p, \mathbf{k}, k_{z}\right)$ : here $p$ is the polarization index, taking the values $p=1,2$, corresponding to TE and TM polarization, respectively, while $\mathbf{k}$ and $k_{z}$ are the transverse and longitudinal components of the wave vector. $H_{\mathrm{at}}$ is the internal Hamiltonian of our two-level atom having ground state $|g\rangle$ and excited state $|e\rangle$ separated by a transition frequency $\omega_{0}$. While $H_{\text {at }}$ is associated with the internal atomic degrees of freedom, the term $H_{\mathrm{Ws}}$ accounts for the external atomic dynamics. The interaction between the atom and the quantum electromagnetic field is written here in the well-known multipolar coupling in dipole approximation [38], where $\boldsymbol{\mu}=q \boldsymbol{\rho}$ (with $q$ being the electron's charge and $\boldsymbol{\rho}$ being the internal atomic coordinate) is the quantum operator associated with the atomic electric dipole moment and the electric field is calculated in the atomic position $\mathbf{r}$.

In order to compute the correction to the complex energy spectrum due to the Casimir-Polder effect, we have used a non-Hermitian equivalent of the complex perturbation theory based on a redefinition of the scalar product, which will be called the $c$-product, defined by [39]

$$
(\varphi \mid \psi)=\int_{-\infty}^{+\infty} d x \varphi(x) \psi(x)
$$


Using this definition, we can define the correction term in the same way as in standard quantum mechanics and obtain the expression of the second-order energy correction,

$$
\Delta E_{n}^{(2)}=\sum_{k \neq n} \frac{\left[\left(\psi_{k}^{(0)}\left|W^{\theta}\right| \psi_{n}^{(0)}\right)\right]^{2}}{E_{n}^{(0)}-E_{k}^{(0)}}
$$

for a complex-scaled Hamiltonian of the form

$$
H^{\theta}=H_{0}^{\theta}+W^{\theta},
$$

where $H_{0}^{\theta}$ is the non-Hermitian unperturbed Hamiltonian and $W^{\theta}$ is a small perturbation. We recall here that the first-order correction in the case of the dipolar approximation for the atom-field interaction is null due to the nature of the atomic dipolar operator $\boldsymbol{\mu}$. After having scaled the Hamiltonian (15), we are now prepared to calculate the complex correction knowing that the unperturbed states can be written as

$$
\left.\left.\mid \psi_{n, 1}^{(0)}\right)=|g\rangle\left|0_{\epsilon}\left(\mathbf{k}, k_{z}\right)\right\rangle \mid n, 1\right),
$$

where $n$ stands for the number of the well where the atom is initially placed (in the first Bloch band). We have to stress here that this state belongs to a product of three spaces and that the transformation (4) acts only on the external atomic states. As a consequence, we will use the c-product only on the external atomic states, whereas we keep the standard scalar product acting on the internal atomic states and field states. These assumptions lead to an expression for the second-order correction of the modified Wannier-Stark energy levels,

$$
\begin{aligned}
\Delta E_{m, 1}^{(2)}= & -\sum_{p} \int_{0}^{+\infty} d k_{z} \\
& \times \int d^{2} \mathbf{k} \sum_{n=1}^{+\infty} \sum_{b=1}^{+\infty} \frac{\left.\left.\left|\left(\psi_{m, 1}^{(0)}\left|H_{\mathrm{int}}^{\theta}\right| e\right\rangle\right| 1_{p}\left(\mathbf{k}, k_{z}\right)\right\rangle \mid n, b\right)\left.\right|^{2}}{E_{n, b}^{(0)}-E_{m, 1}^{(0)}+\hbar\left(\omega+\omega_{0}\right)},
\end{aligned}
$$

where

$$
H_{\mathrm{int}}^{\theta}=-\boldsymbol{\mu} \cdot \mathbf{E}\left(\mathbf{r} e^{i \theta}\right) .
$$

Equation (20) can be simplified considering that in a shallow trap, as in the case of FORCA-G, the Bloch bands above the second one are not trapped in the lattice, so that the wave functions associated with these bands are very delocalized and the overlap with the states of the first band is very weak. This observation allows us to restrict the sum over $b$ to its first two terms. Moreover, due to the extension of the modified WannierStark wave functions in the first band, it is sufficient to take into account only 20 terms in the sum over $n$. Finally, the weakness of the imaginary part of the modified Wannier-Stark states as well as those of the difference of the real part of the WannierStark spectrum with respect to the term $\hbar\left(\omega+\omega_{0}\right)$ allows us to ignore the contribution $E_{n, b}^{(0)}-E_{m, 1}^{(0)}$ in the denominator in Eq. (20). All these simplifications lead to a new expression of the correction,

$$
\begin{aligned}
\Delta E_{m, 1}^{(2)}= & \int_{0}^{\infty} d z \int_{0}^{\infty} d z^{\prime} \sum_{n=m-10}^{m+10}\left\{-\sum_{p} \int_{0}^{+\infty} d k_{z} \int d^{2} \mathbf{k}\left[\left(\frac{(m, 1 \mid z)(z \mid n, 1)\left(m, 1 \mid z^{\prime}\right)\left(z^{\prime} \mid n, 1\right)}{\hbar\left(\omega+\omega_{0}\right)}\right.\right.\right. \\
& \left.\left.\left.+\frac{(m, 1 \mid z)(z \mid n, 2)\left(m, 1 \mid z^{\prime}\right)\left(z^{\prime} \mid n, 2\right)}{\hbar\left(\omega+\omega_{0}\right)}\right) A_{p}^{(\theta)}\left(\mathbf{k}, k_{z}, \mathbf{r}\right) A_{p}^{(\theta) \dagger}\left(\mathbf{k}, k_{z}, \mathbf{r}^{\prime}\right)\right]\right\} \\
= & -\int_{0}^{\infty} d z \int_{0}^{\infty} d z^{\prime} \sum_{p} \int_{0}^{+\infty} d k_{z} \\
& \times \int d^{2} \mathbf{k} \sum_{n=m-10}^{m+10}\left\{\frac{W S_{m, 1}(z) W S_{m, 1}\left(z^{\prime}\right)}{\hbar\left(\omega+\omega_{0}\right)} A_{p}^{(\theta)}\left(\mathbf{k}, k_{z}, \mathbf{r}\right) A_{p}^{(\theta) \dagger}\left(\mathbf{k}, k_{z}, \mathbf{r}^{\prime}\right)\left[W S_{n, 1}(z) W S_{n, 1}\left(z^{\prime}\right)+W S_{n, 2}(z) W S_{n, 2}\left(z^{\prime}\right)\right]\right\}
\end{aligned}
$$

where $W S_{n, m}(x)$ is the state for the modified Wannier-Stark states taking into account the presence of the surface and

$$
A_{p}^{(\theta)}\left(\mathbf{k}, k_{z}, \mathbf{r}\right)=-\left\langle 0_{p}\left(\mathbf{k}, k_{z}\right)\left|\left\langle g\left|\boldsymbol{\mu} \cdot \boldsymbol{E}\left(\mathbf{r} e^{i \theta}\right)\right| e\right\rangle\right| 1_{p}\left(\mathbf{k}, k_{z}\right)\right\rangle=-\frac{i}{\pi} \sqrt{\frac{\hbar \omega}{4 \pi \varepsilon_{0}}} e^{i \mathbf{k} \cdot \boldsymbol{\rho} e^{i \theta}} \boldsymbol{\mu}_{e g} \cdot \mathbf{f}_{p}\left(\mathbf{k}, k_{z}, z e^{i \theta}\right),
$$

where $\mathbf{f}_{p}\left(\mathbf{k}, k_{z}, z\right)$ are the mode functions of the electric field in the presence of a perfectly conducting mirror in $z=0$ [40],

$$
\mathbf{f}_{1}\left(\mathbf{k}, k_{z}, z\right)=\hat{\mathbf{k}} \times \hat{\mathbf{z}} \sin \left(k_{z} z\right), \quad \mathbf{f}_{2}\left(\mathbf{k}, k_{z}, z\right)=\hat{\mathbf{k}} \frac{i c k_{z}}{\omega} \sin \left(k_{z} z\right)-\hat{\mathbf{z}} \frac{c k}{\omega} \cos \left(k_{z} z\right),
$$

with $\hat{\mathbf{k}}=\mathbf{k} / k$ and $\hat{\mathbf{z}}=(0,0,1)$. Unfortunately, the terms $A_{p}^{(\theta)}\left(\mathbf{k}, k_{z}, \mathbf{r}\right)$ and $A_{p}^{(\theta) \dagger}\left(\mathbf{k}, k_{z}, \mathbf{r}^{\prime}\right)$ lead to a divergent integral when the coordinate $z$ is complex scaled. Indeed, using the definitions of the electric field given in [19], we obtain the following divergent expression for the correction:

$$
\begin{aligned}
\Delta E_{m, 1}^{(2)}= & -\frac{\hbar \boldsymbol{\mu}_{e g}^{2}}{4 \pi^{3} \epsilon_{0}} \int_{0}^{\infty} d z \int_{0}^{\infty} d z^{\prime} \int_{0}^{+\infty} d k_{z} \int d^{2} \mathbf{k} \sum_{n=m-10}^{m+10}\left(\frac{W_{m, 1}(z) W_{m, 1}\left(z^{\prime}\right)}{\hbar\left(\omega+\omega_{0}\right)}\left[W_{n, 1}(z) W_{n, 1}\left(z^{\prime}\right)+W_{n, 2}(z) W_{n, 2}\left(z^{\prime}\right)\right]\right. \\
& \left.\times \omega\left\{\left[1+\left(\frac{c k_{z}}{\omega}\right)^{2}\right] \sin \left(k_{z} z e^{i \theta}\right) \sin \left(k_{z} z^{\prime} e^{i \theta}\right)+\left(\frac{c k}{\omega}\right)^{2} \cos \left(k_{z} z e^{i \theta}\right) \cos \left(k_{z} z^{\prime} e^{i \theta}\right)\right\}\right) .
\end{aligned}
$$


This divergence is fundamental because the terms in cosh and sinh (coming from the sin and cos functions with complex arguments) produce a divergence which is not sensitive to any ordinary regularization technique. This could be due to the fact that the atom is treated all along the calculation as a pointlike particle, whereas its finite size should be taken into account. However, this effect is difficult to characterize because the scaling of the Hamiltonian makes the coordinate representing the atom-wall distance complex so that the atomic size should be defined as a complex quantity, which is far from natural. So, for the time being, the calculation of the effect of the Casimir-Polder interaction on the lifetimes of the atomic states remains an open problem.

\section{CONCLUSIONS}

In this paper we have studied the modifications to the lifetime of atoms trapped in an optical lattice in proximity of a surface. We have shown that the boundary condition introduced by the presence of the surface only marginally modifies the value of the ordinary Wannier-Stark lifetimes, leaving them almost infinite with respect to the duration of a measurement in a typical atomic-interferometry experiment such as the recently proposed FORCA-G. The same holds for the presence of a hypothetical Yukawa deviation from Newton's gravitational law. In our analysis, we have modeled the surface as both an infinite and a finite potential barrier and considered an atom both above and below the surface.

As a natural development of our work, it could be interesting to investigate the behavior of the states in front of a more realistic surface, abandoning the assumptions of perfect conduction and infinite extension. This is the subject of ongoing work and will be part of a future presentation. As discussed in the last section, another problem which remains open is the precise calculation of the perturbation of the lifetimes due to the Casimir-Polder effect. However, we expect that this will not significantly reduce the lifetime. On the contrary, the Casimir-Polder force is attractive, so for atoms below the surface it will tend to counteract Landau-Zener tunneling, thus increasing the lifetime, rather than decreasing it. This assumption is made knowing that the Casimir-Polder potential is attractive toward the surface, whereas the Landau-Zener effect tends to drag the atom away from the surface. Thus at the moment we can assume that the Casimir-Polder effect should act on the lifetimes in an opposite way with respect to the Landau-Zener effect, so it should increase the lifetime of the atomic states. Finally, we point out that in this work we have not taken into account chemical processes between the atoms and the surface (atoms "sticking" to the surface) as we have considered the surface to be a simple potential barrier. A more realistic analysis should include an additional potential describing that interaction but is beyond the scope of the present paper.

\section{ACKNOWLEDGMENTS}

This research was carried out within the project iSense, which acknowledges the financial support of the Future and Emerging Technologies (FET) program within the Seventh Framework Programme for Research of the European Commission, under FET-Open Grant No. 250072. We also gratefully acknowledge support by Ville de Paris [Emergence(s) program] and IFRAF. The authors thank N. Moiseyev, H. J. Korsch, Q. Beaufils, A. Hilico, B. Pelle, and F. Pereira dos Santos for fruitful and stimulating discussions.
[1] N. W. Ashcroft and N. D. Mermin, Solid State Physics (Holt, Rinehart and Winston, New York, 1976).

[2] G. H. Wannier, Phys. Rev. 117, 432 (1960).

[3] N. Moiseyev, Phys. Rep. 302, 211 (1998).

[4] M. Gluck, A. R. Kolovsky, H. J. Korsch, and N. Moiseyev, Eur. Phys. J. D 4, 239 (1998).

[5] M. Gluck, A. R. Kolovsky, and H. J. Korsch, Phys. Rep. 366, 103 (2002).

[6] S. Wimberger, P. Schlagheck, and R. Mannella, J. Phys. B 39, 729 (2006).

[7] S. Wimberger, R. Mannella, O. Morsch, E. Arimondo, A. R. Kolovsky, and A. Buchleitner, Phys. Rev. A 72, 063610 (2005).

[8] C. Sias, A. Zenesini, H. Lignier, S. Wimberger, D. Ciampini, O. Morsch, and E. Arimondo, Phys. Rev. Lett. 98, 120403 (2007).

[9] A. Zenesini, H. Lignier, G. Tayebirad, J. Radogostowicz, D. Ciampini, R. Mannella, S. Wimberger, O. Morsch, and E. Arimondo, Phys. Rev. Lett. 103, 090403 (2009).

[10] G. Tayebirad, A. Zenesini, D. Ciampini, R. Mannella, O. Morsch, E. Arimondo, N. Lörch, and S. Wimberger, Phys. Rev. A 82, 013633 (2010).
[11] N. Lörch, F. V. Pepe, H. Lignier, D. Ciampini, R. Mannella, O. Morsch, E. Arimondo, P. Facchi, G. Florio, S. Pascazio, and S. Wimberger, Phys. Rev. A 85, 053602 (2012).

[12] D. Witthaut, E. M. Graefe, S. Wimberger, and H. J. Korsch, Phys. Rev. A 75, 013617 (2007).

[13] K. Rapedius, C. Elsen, D. Witthaut, S. Wimberger, and H. J. Korsch, Phys. Rev. A 82, 063601 (2010).

[14] L. D. Landau, Phys. Z. Sowjetunion 2, 46 (1932).

[15] C. Zener, Proc. R. Soc. London, Ser. A 137, 696 (1932).

[16] C. Zener, Proc. R. Soc. London, Ser. A 145, 523 (1934).

[17] P. Lemonde and P. Wolf, Phys. Rev. A 72, 033409 (2005).

[18] P. Wolf, P. Lemonde, A. Lambrecht, S. Bize, A. Landragin, and A. Clairon, Phys. Rev. A 75, 063608 (2007).

[19] R. Messina, S. Pelisson, M.-C. Angonin, and P. Wolf, Phys. Rev. A 83, 052111 (2011).

[20] Q. Beaufils, G. Tackmann, X. Wang, B. Pelle, S. Pelisson, P. Wolf, and F. P. dos Santos, Phys. Rev. Lett. 106, 213002 (2011).

[21] G. Tackmann, B. Pelle, A. Hilico, Q. Beaufils, and F. Pereira dos Santos, Phys. Rev. A 84, 063422 (2011).

[22] S. Pelisson, R. Messina, M.-C. Angonin, and P. Wolf, Phys. Rev. A 86, 013614 (2012). 
[23] B. Pelle, A. Hilico, G. Tackmann, Q. Beaufils, and F. Pereira dos Santos, Phys. Rev. A 87, 023601 (2013).

[24] J. Agler and R. Froese, Commun. Math. Phys. 100, 161 (1985).

[25] J. E. Avron, Phys. Rev. Lett. 37, 1568 (1976).

[26] F. Bentosela, V. Grecchi, and F. Zironi, J. Phys. C 15, 7119 (1982).

[27] V. Grecchi, M. Maioli, and A. Sacchetti, J. Phys. A 26, L379 (1993).

[28] V. Grecchi and A. Sacchetti, Commun. Math. Phys. 185, 359 (1997).

[29] V. Buslaev and A. Grigis, J. Math. Phys. 39, 2520 (1998).

[30] S. Glutsch and F. Bechstedt, Phys. Rev. B 60, 16584 (1999).

[31] W. P. Reinhardt, Annu. Rev. Phys. Chem. 33, 223 (1982).

[32] A. Buchleitner, B. Grémaud, and D. Delande, J. Phys. B 27, 2663 (1994).

[33] E. Balslev and J. M. Combes, Commun. Math. Phys. 22, 280 (1971).
[34] W. H. Press, S. A. Teukolsky, W. T. Vetterling, and B. P. Flannery, Numerical Recipes in C: The Art of Scientific Computing (Cambridge University Press, Cambridge, 1992).

[35] Q. Niu, X.-G. Zhao, G. A. Georgakis, and M. G. Raizen, Phys. Rev. Lett. 76, 4504 (1996).

[36] E. Anderson, Z. Bai, C. Bischof, S. Blackford, J. Demmel, J. Dongarra, J. Du Croz, A. Greenbaum, S. Hammmerling, A. McKenney, and D. Sorensen, LAPACK User' Guide, 3rd ed. (Society for Industrial and Applied Mathematics, Philadelphia, 1999).

[37] A. A. Geraci, S. B. Papp, and J. Kitching, Phys. Rev. Lett. 105, 101101 (2010).

[38] E. Power and S. Zienau, Philos. Trans. R. Soc. London, Ser. A 251, 427 (1959).

[39] N. Moiseyev, Non-Hermitian Quantum Mechanics (Cambridge University Press, Cambridge, 2011).

[40] G. Barton, J. Phys. B 7, 2134 (1974). 